TRANSACTIONS OF THE

AMERICAN MATHEMATICAL SOCIETY

Volume 365, Number 2, February 2013, Pages 555-578

S 0002-9947(2012)05868-5

Article electronically published on September 24, 2012

\title{
GROUPS ACTING ON SEMIMETRIC SPACES AND QUASI-ISOMETRIES OF MONOIDS
}

\author{
ROBERT GRAY AND MARK KAMBITES
}

\begin{abstract}
We study groups acting by length-preserving transformations on spaces equipped with asymmetric, partially-defined distance functions. We introduce a natural notion of quasi-isometry for such spaces and exhibit an extension of the Švarc-Milnor lemma to this setting. Among the most natural examples of these spaces are finitely generated monoids and semigroups and their Cayley and Schützenberger graphs. We apply our results to show that a number of important properties of monoids are quasi-isometry invariants.
\end{abstract}

\section{INTRODUCTION}

One of the most exciting and influential developments of 20th century mathematics was the advent of geometric group theory. A key concept in this subject is quasi-isometry: a notion of equivalence between metric spaces which formally captures the intuitive idea of two spaces looking the same "when viewed from far away". The Švarc-Milnor lemma (which has been described as the "fundamental observation of geometric group theory" [8]) guarantees that a discrete group which acts in a suitably controlled way upon a geodesic metric space is quasi-isometric to that space. This fact establishes a deep connection between geometry and group theory, which continues to inform our understanding of both subjects.

At the same time, another pervasive theme in modern mathematics has been the discovery of applications for traditionally pure areas of mathematics; this trend is typified by the emergence of such fields as algebraic automata theory [1, 10, 11. and tropical geometry 30. Real-world problems often do not display the high degrees of symmetry and structure enjoyed by many of the objects of classical pure mathematics, and this has increasingly led pure mathematicians to study fundamentally non-rigid, asymmetric structures, such as semigroups (which arise in algebraic automata theory) and semirings (which arise in tropical geometry). Another key example is that of spaces with asymmetric distance functions. These have traditionally been viewed as the poor relations of their symmetric counterparts,

Received by the editors August 25, 2009.

2010 Mathematics Subject Classification. Primary 20M05, 20M30, 05C20.

Key words and phrases. Monoid, group, finitely generated, action, semimetric space, quasimetric space.

The first author's research was supported by an EPSRC Postdoctoral Fellowship.

The second author's research was supported by an RCUK Academic Fellowship. The second author gratefully acknowledges the support of the Centre for Interdisciplinary Research in Computational Algebra during a visit to St Andrews.

(C) 2012 American Mathematical Society Reverts to public domain 28 years from publication 
but it is readily apparent that they arise with great frequency in nature, and hence also in applied mathematics, and they now also seem increasingly relevant in pure mathematics.

The paper explores a connection between these two trends in mathematics by studying discrete groups acting by isometries on semimetric spaces, by which we mean spaces equipped with asymmetric, partially defined distance functions. It transpires that both the notion of quasi-isometry and the Svarc-Milnor lemma itself admit natural and straightforward extensions to the more general setting of groups acting on semimetric spaces. One area of pure mathematics where asymmetric distance functions naturally arise is the theory of finitely generated semigroups and monoids; indeed, any finitely generated monoid or semigroup is naturally endowed with the structure of semimetric space. We show that a number of natural and widely studied properties of semigroups and monoids are invariant under quasiisometry. Some of these are obtained as applications of our results concerning group actions, while others are proved directly by geometric arguments.

In addition to this introduction, this article comprises eight sections. In Section 2 we introduce quasi-isometries between semimetric spaces, and some of their foundational properties. In Section 3 we consider groups acting by isometries on semimetric spaces, establishing an analogue of the Švarc-Milnor lemma. Section 4 studies some important examples of semimetric spaces which arise from directed graphs, and in particular from finitely generated semigroups and monoids. Section 5 demonstrates how group actions on semimetric spaces can be applied in semigroup theory by establishing that a number of natural properties of finitely generated monoids are invariant under quasi-isometry. Finally, Sections [6] and 7 contain direct (without reliance on group actions) geometric proofs that some further important properties of finitely generated monoids (namely growth rate and number of ends) are also quasi-isometry invariants.

\section{Semimetric and Quasi-metric spaces}

In this section we introduce the main objects of our study, namely spaces equipped with asymmetric partially-defined distance functions, and prove a number of foundational results.

We begin by discussing some issues relating to terminology. There are several natural ways to generalise the notion of a metric space by weakening the axioms; these have arisen in numerous different contexts, and terminology for them is not standardised. Spaces with asymmetric distance functions are perhaps most widely called "quasi-metric" spaces (see for example [24, 42]). Unfortunately, this terminology conflicts fundamentally with the standard language of geometric group theory, where the prefix "quasi" is by convention used to mean "up to finite additive and multiplicative distortion". To complicate matters still further, we shall actually need to study spaces which are metric up to finite distortion, that is, "quasi-metric" in the sense that group theorists would expect. Since we place minimal reliance on the existing theory, we have chosen to develop terminology consistent with that of geometric group theory.

Throughout this paper we write $\mathbb{R}^{\infty}$ for the set $\mathbb{R}^{\geq 0} \cup\{\infty\}$ of non-negative real numbers with $\infty$ adjoined. We extend the usual ordering on $\mathbb{R}^{\geq 0}$ to $\mathbb{R}^{\infty}$ in the obvious way, taking in particular $\infty$ to be the infimum value of the empty set, and the supremum value of any subset of $\mathbb{R}^{\geq 0}$ which is not bounded above. We also 
extend addition of non-negative reals to $\mathbb{R}^{\infty}$ and multiplication of positive reals to $\mathbb{R}^{\infty} \backslash\{0\}$ by defining

$$
\infty+x=x+\infty=y \infty=\infty y=\infty
$$

for all $x \in \mathbb{R}^{\infty}$ and $y \in \mathbb{R}^{\infty} \backslash\{0\}$.

Definition 1 (Semimetric space). A semimetric space is a pair $(X, d)$ where $X$ is a set and $d: X \times X \rightarrow \mathbb{R}^{\infty}$ is a function satisfying:

(i) $d(x, y)=0$ if and only if $x=y$ and

(ii) $d(x, z) \leq d(x, y)+d(y, z)$

for all $x, y, z \in X$. If, in addition, $d$ satisfies $d(x, y)=d(y, x)$, then we say that $(X, d)$ is a metric space.

A point $x_{0} \in X$ is called a basepoint for the space $X$ if $d\left(x_{0}, y\right) \neq \infty$ for all $y \in X$. The space is called strongly connected if every point is a basepoint, that is, if no two points are at distance $\infty$.

Definition 2 (Isometric embeddings). A map $f: X \rightarrow X^{\prime}$ between semimetric spaces is called an isometric embedding if

$$
d(f(x), f(y))=d(x, y) \text { for all } x, y \in X
$$

and is called an isometry if it is a surjective isometric embedding.

Definition 3 (Paths and geodesics). Let $X$ be a semimetric space, $x, y \in X$ and $n \in \mathbb{R}^{\geq 0}$. A path of length $n$ from $x$ to $y$ is a map $p:[0, n] \rightarrow X$ such that $p(0)=x$, $p(n)=y$ and $d(p(a), p(b)) \leq b-a$ for all $0 \leq a \leq b \leq n$.

If $d(x, y) \neq \infty$, then a geodesic from $x$ to $y$ is a path of length $d(x, y)$ from $x$ to $y$. The semimetric space $X$ is called geodesic if for all $x, y \in X$ with $d(x, y) \neq \infty$ there exists at least one geodesic from $x$ to $y$.

Notice that if $p:[0, d(x, y)] \rightarrow X$ is a geodesic from $x$ to $y$, then for any $a \in$ $[0, d(x, y)]$ we have $d(x, p(a)) \leq a$ and $d(p(a), y) \leq d(x, y)-a$. But by the triangle inequality $d(x, y) \leq d(x, p(a))+d(p(a), y)$ and distances are positive, so we deduce that $d(x, p(a))=a$ and $d(p(a), y)=d(x, y)-a$.

However, a geodesic cannot be defined as an isometric embedding of a particular space, in the same way that a geodesic in a conventional metric space is an isometric embedding of an interval on the real line. The requirements for a function to be a geodesic place demands on distances in only one direction; distance in the other direction may vary, so images of geodesics of the same length need not be isometric.

Definition 4 (Distance between sets). Let $X$ be a semimetric space and $A, B \subseteq X$. Then we define the distance from $A$ to $B$ to be

$$
d(A, B)=\inf _{a \in A, b \in B} d(a, b) .
$$

Note in particular that two sets are at distance $\infty$ if and only if all their members are at distance $\infty$.

Definition 5 (Balls). Let $x_{0} \in X$ and let $r$ be a non-negative real number. The out-ball of radius $r$ based at $x_{0}$ is

$$
\overrightarrow{\mathcal{B}}_{r}\left(x_{0}\right)=\left\{y \in X: d\left(x_{0}, y\right) \leq r\right\} .
$$

Dually, the in-ball of radius $r$ based at $x_{0}$ is defined by

$$
\overleftarrow{\mathcal{B}}_{r}\left(x_{0}\right)=\left\{y \in X: d\left(y, x_{0}\right) \leq r\right\}
$$


and the strong ball of radius $r$ based at $x_{0}$ is

$$
\mathcal{B}_{r}\left(x_{0}\right)=\overrightarrow{\mathcal{B}}_{r}\left(x_{0}\right) \cap \overleftarrow{\mathcal{B}}_{r}\left(x_{0}\right)
$$

Definition 6 (Quasi-dense). Let $T$ be a subset of a semimetric space $X=(X, d)$, and $0 \leq \mu<\infty$. We say that $T$ is $\mu$-quasi-dense in $X$ if for all $x \in X$ there exists $y \in T$ such that

$$
\max (d(y, x), d(x, y)) \leq \mu,
$$

that is, if $X$ is covered by strong balls of radius $\mu$ around points in $T$. A subset is called quasi-dense if it is $\mu$-quasi-dense for some $0 \leq \mu<\infty$.

Definition 7 (Quasi-isometric embedding and quasi-isometry). Let $f:(X, d) \rightarrow$ $\left(X^{\prime}, d^{\prime}\right)$ be a map between semimetric spaces, and let $1 \leq \lambda<\infty$ and $0<\epsilon<\infty$ be constants. We say that $f$ is a $(\lambda, \epsilon)$-quasi-isometric embedding and $X$ embeds quasi-isometrically in $X^{\prime}$ if

$$
\frac{1}{\lambda} d(x, y)-\epsilon \leq d^{\prime}(f(x), f(y)) \leq \lambda d(x, y)+\epsilon
$$

for all $x, y \in X$. If in addition the image $f(X)$ of $f$ is $\mu$-quasi-dense with constant $\mu$, then $f$ is called a $(\lambda, \epsilon, \mu)$-quasi-isometry, and we say that $X$ and $Y$ are quasiisometric.

The following straightforward proposition can be proved exactly as in the case of quasi-isometries of metric spaces (see, for example, [14, Exercise 10.6]).

Proposition 1. Quasi-isometric embedding is a reflexive and transitive relation on the class of semimetric spaces. Quasi-isometry is an equivalence relation on the class of semimetric spaces.

Note that any isometric embedding is a quasi-isometric embedding; in particular, the inclusion map from a subspace into its containing space is always a quasiisometric embedding and is a quasi-isometry exactly if the subspace is quasi-dense.

Definition 8 (Quasi-metric space). Let $1 \leq \lambda<\infty$ and $0 \leq \epsilon<\infty$. A semimetric space $X$ is called $(\lambda, \epsilon)$-quasi-metric if it is strongly connected and $d(y, x) \leq$ $\lambda d(x, y)+\epsilon$ for all $x, y \in X$. A semimetric space is called quasi-metric if it is $(\lambda, \epsilon)$-quasi-metric for some $\lambda$ and $\epsilon$.

Notice that if a non-empty semimetric space admits $\lambda$ and $\epsilon$ such that the inequalities in Definition 8 are satisfied, then it is strongly connected (and hence quasi-metric) if and only if it has a basepoint. Intuitively, a quasi-metric space is a semimetric space in which the distances between vertices do not depend too dramatically on the direction in which one travels between them. One might expect such a space to behave rather like a metric space; in fact, the following easy proposition says that it must resemble some particular metric space.

Proposition 2. A semimetric space $X$ is quasi-metric if and only if it is quasiisometric to a metric space.

Proof. Suppose first that $(X, d)$ is a $(\lambda, \epsilon)$-quasi-metric space. Define $d^{\prime}: X \times X \rightarrow$ $\mathbb{R}^{\geq 0}$ by $d^{\prime}(x, y)=d(x, y)+d(y, x)$, noting that $d(x, y)+d(y, x)$ is indeed finite since $(X, d)$ is strongly connected. It is well known and easy to prove that $\left(X, d^{\prime}\right)$ is a metric space. We claim that the identity map $f: X \rightarrow X$ is a $\left(\lambda^{\prime}, \epsilon, 0\right)$-quasiisometry where $\lambda^{\prime}=\max \left(\lambda+1, \frac{\lambda}{\lambda+1}\right)$ (note that $\lambda^{\prime} \geq 1$ ). 
Since $f$ is surjective, its image is 0 -quasi-dense. Now for $x, y \in X$ we have

$$
\begin{aligned}
d^{\prime}(f(x), f(y)) & =d^{\prime}(x, y) \\
& =d(x, y)+d(y, x) \\
\leq & d(x, y)+(\lambda d(x, y)+\epsilon) \\
& =(\lambda+1) d(x, y)+\epsilon \\
& \leq \lambda^{\prime} d(x, y)+\epsilon .
\end{aligned}
$$

Also, since $(X, d)$ is $(\lambda, \epsilon)$-quasi-metric we have

$$
\begin{aligned}
d^{\prime}(f(x), f(y)) & =d(x, y)+d(y, x) \\
& \geq d(x, y)+\left(\frac{1}{\lambda} d(x, y)-\epsilon\right) \\
& =\left(\frac{\lambda+1}{\lambda}\right) d(x, y)-\epsilon \\
& \geq \frac{1}{\lambda^{\prime}} d(x, y)-\epsilon .
\end{aligned}
$$

For the converse, suppose that $f: X \rightarrow X^{\prime}$ is a $(\lambda, \epsilon, \mu)$-quasi-isometry where $\left(X^{\prime}, d^{\prime}\right)$ is a metric space. Let $x, y \in X$ be arbitrary. Then since $\left(X^{\prime}, d^{\prime}\right)$ is metric we have

$$
\frac{1}{\lambda} d(x, y)-\epsilon \leq d^{\prime}(f(x), f(y))=d^{\prime}(f(y), f(x)) \leq \lambda d(y, x)+\epsilon,
$$

which implies

$$
d(x, y) \leq \lambda(\lambda d(y, x)+\epsilon+\epsilon)=\lambda^{2} d(y, x)+2 \lambda \epsilon .
$$

So $X$ is $\left(\lambda^{2}, 2 \lambda \epsilon\right)$-quasi-metric.

Corollary 1. Quasi-metricity is a quasi-isometry invariant.

An instructive example of a quasi-metric space which is not metric is a finitely generated group equipped with the word metric induced by a non-symmetric finite monoid generating set. We shall see below (Theorem 1) that a semimetric space which admits a suitably controlled action by a group must also be quasi-metric.

\section{Groups aCting on SEMimetriC SPACES}

In this section we consider groups acting by isometries on semimetric spaces and prove an analogue of the Švarc-Milnor lemma. As we shall see in Section 5, such actions arise naturally in semigroup theory.

Let $G$ be a group acting by isometries on the left of a semimetric space $X$. We write $g x$ for the image of $x \in X$ under the action of $g \in G$.

Definition 9 (Cocompact action). An action by isometries of a group $G$ on a semimetric space $X$ is called cocompact if there is a strong ball $B$ of finite radius such that $\{g B: g \in G\}$ covers $X$.

We remark that, while it is usual to define a cocompact action in purely topological terms (using compact sets), our definition relies on the semimetric (using balls of finite radius). This is inevitable, since we have no clear topology underlying our semimetric spaces, and hence no abstract notion of a compact set. However, in the event that $X$ is a proper metric space (one in which closed balls of finite radius are 
compact in the topology induced by the metric) it is easily seen that our notion of a cocompact action coincides with the usual one.

Lemma 1. Let $G$ be a group acting by isometries on a semimetric space $(X, d)$. Then the following are equivalent:

(i) for every out-ball $B$ of finite radius the set $\{g \in G: d(B, g B)=0\}$ is finite;

(ii) for every out-ball $B$ of finite radius the set $\{g \in G: d(g B, B)=0\}$ is finite;

(iii) for every out-ball $B$ of finite radius the set $\{g \in G: B \cap g B \neq \varnothing\}$ is finite.

The analogous statement also holds for in-balls.

Proof. We shall prove the result for out-balls. The result for in-balls may be dealt with using similar arguments.

Let $B=\overrightarrow{\mathcal{B}}_{r}\left(x_{0}\right)$ be an out-ball and let $g \in G$. If $B \cap g B \neq \varnothing$, then $d(B, g B)=$ $d(g B, B)=0$. This shows (i) $\Rightarrow$ (iii) and (ii) $\Rightarrow$ (iii). To see (iii) $\Rightarrow$ (i) suppose that $d(B, g B)=0$. Set $C=\overrightarrow{\mathcal{B}}_{r+\epsilon}\left(x_{0}\right)$ for some fixed $\epsilon>0$. Then there exist $x \in B$ and $y \in g B$ with $d(x, y)<\epsilon$ which implies $y \in C \cap g B \subseteq C \cap g C$, and hence $C \cap g C \neq \varnothing$. Therefore

$$
\{g \in G: d(B, g B)=0\} \subseteq\{g \in G: C \cap g C \neq \varnothing\},
$$

which is finite by (iii). The proof that (iii) implies (ii) is similar.

Definition 10 (Proper action). We say that $G$ is acting outward properly (respectively inward properly) on $X$ if one of the equivalent conditions given in Lemma 1 holds.

Just as for cocompact actions, our definitions of inward/outward proper inevitably rely on the semimetric, where the usual notion of a proper action is defined purely topologically. Again, provided $X$ is a proper metric space, our notions of inward proper and outward proper coincide with the usual definition of proper (and hence with each other).

In the usual way we regard a finitely generated group as a metric space via its Cayley graph. The quasi-isometry class of a finitely generated group is well defined since Cayley graphs with respect to different finite generating sets are quasiisometric. More generally, this is true for finitely generated semigroups, as we shall see in Section 4 .

We now prove the main result of this section which is a Švarc-Milnor lemma for groups acting on semimetric spaces.

Theorem 1. Let $G$ be a group acting outward properly and cocompactly by isometries on a geodesic semimetric space $X$ with basepoint. Then $G$ is a finitely generated group quasi-isometric to $X$. In particular, $X$ is a quasi-metric space.

Proof. Since $G$ is acting cocompactly there is a strong ball $B$, based at $x_{0}$ say, of radius $R$ such that $(g B)_{g \in G}$ covers $X$. Now $X$ contains a basepoint ( $b$ say), which must lie in $g B$ for some $g \in G$. Since the action is by isometries, it follows that $g^{-1} b$ is a basepoint in $B$, and since $B$ is strongly connected, we deduce that $x_{0}$ is also a basepoint. Now let

$$
S=\{g \in G: d(B, g B)=0\} .
$$


Note that $S$ is finite, since it is contained in $\left\{g \in G: d\left(\overrightarrow{\mathcal{B}}_{R}\left(x_{0}\right), g \overrightarrow{\mathcal{B}}_{R}\left(x_{0}\right)\right)=0\right\}$ and the action of $G$ is outward proper. Clearly $e \in S$, where $e$ denotes the identity element of $G$ (but it is not necessarily the case that $S$ is closed under the taking of inverses).

Let $C=\overrightarrow{\mathcal{B}}_{5 R}\left(x_{0}\right)$, noting that $B \subseteq C$, and define

$$
Q=\{g B: d(B, g B) \neq 0 \text { and } d(C, g B)=0\} .
$$

Note that $Q$ is finite, since it is contained in $\{g B: d(C, g C)=0\}$, which is finite since the action is outward proper. Hence, we may choose a positive real number $r$ such that $r<R$ and $r<d(B, g B)$ for every $g B \in Q$.

We claim $r$ has the property that for all $h \in G$, if $d(B, h B)<r$, then $d(B, h B)$ $=0$. To see this, suppose on the contrary that $d(B, h B)<r$ but that $d(B, h B) \neq 0$. Since $d(B, h B)<r$ there exist $u \in B$ and $v \in h B$ with $d(u, v)<r$. Since $u \in B$ we have $d\left(x_{0}, u\right) \leq R$. Therefore

$$
d\left(x_{0}, v\right) \leq d\left(x_{0}, u\right)+d(u, v)<R+r \leq 2 R<5 R
$$

so that $v \in C=\overrightarrow{\mathcal{B}}_{5 R}\left(x_{0}\right)$. Thus $v \in C \cap h B$ so $d(C, h B)=0$ and $h B \in Q$. By the choice of $r$ it follows that $r<d(B, h B)$. But this contradicts the assumption that $d(B, h B)<r$, and so proves the claim.

Now choose a positive real number $l<r$. We claim that $S$ generates $G$ and that for all $g \in G$

$$
d_{S}(e, g) \leq \frac{1}{l} d\left(x_{0}, g x_{0}\right)+1 .
$$

To see this, let $g \in G$ be arbitrary. Since the semimetric space $X$ is geodesic and $x_{0}$ is a basepoint, we may choose points $x_{1}, x_{2}, \ldots, x_{k+1}=g x_{0}$ such that $d\left(x_{i}, x_{i+1}\right)=l$ for $0 \leq i<k$ and $d\left(x_{k}, x_{k+1}\right)=l^{\prime} \leq l$. Define $g_{0}=e, g_{k+1}=g$, and for $1 \leq i \leq k$ choose $g_{i} \in G$ such that $x_{i} \in g_{i} B$; such choices are possible since $(g B)_{g \in G}$ covers $X$. Now for $0 \leq i \leq k$ set $s_{i}=g_{i}^{-1} g_{i+1}$ and observe that

$$
g=g_{0} g_{0}^{-1} g_{1} g_{1}^{-1} g_{2} g_{2}^{-1} \ldots g_{k} g_{k}^{-1} g_{k+1}=e s_{0} s_{1} \ldots s_{k} .
$$

Since $d\left(x_{i}, x_{i+1}\right) \leq l<r, x_{i} \in g_{i} B$, and $x_{i+1} \in g_{i+1} B$ it follows that $d\left(g_{i} B, g_{i+1} B\right)$ $<r$ which, since the group is acting by isometries, yields $d\left(B, g_{i}^{-1} g_{i+1} B\right)<r$. By the above claim we conclude that $d\left(B, g_{i}^{-1} g_{i+1} B\right)=0$, and thus $s_{i}=g_{i}^{-1} g_{i+1} \in S$. This proves that $S$ generates the group $G$.

Now $d_{S}(e, g) \leq k+1$, since we have written $g$ as a product of $k+1$ generators, and $k l=d\left(x_{0}, g x_{0}\right)-l^{\prime}$, where $l^{\prime} \leq l$. So

$$
d_{S}(e, g) \leq k+1=\frac{1}{l} d\left(x_{0}, g x_{0}\right)+\left(1-\frac{l^{\prime}}{l}\right) \leq \frac{1}{l} d\left(x_{0}, g x_{0}\right)+1 .
$$

Conversely, an easy inductive argument shows that for all $g \in G$, we have $d\left(x_{0}, g x_{0}\right) \leq \lambda d_{S}(e, g)$ for all $g \in G$, where $\lambda=\max \left\{d\left(x_{0}, s x_{0}\right) \mid s \in S\right\}$.

Now consider the mapping $f: G \rightarrow X$ defined by $g \mapsto g x_{0}$. It follows from the observations above that

$$
d_{S}\left(g_{1}, g_{2}\right) \leq \frac{1}{l} d\left(f\left(g_{1}\right), f\left(g_{2}\right)\right)+1
$$


and also

$$
d\left(f\left(g_{1}\right), f\left(g_{2}\right)\right) \leq \lambda d_{S}\left(g_{1}, g_{2}\right)
$$

for all $g_{1}, g_{2} \in G$. Moreover, given $x \in X$, since $(\alpha B)_{\alpha \in G}$ covers $X$ we conclude that there exists $h \in G$ with $x \in h B$, and thus

$$
\max (d(f(h), x), d(x, f(h))) \leq R .
$$

Hence $G$ and $X$ are quasi-isometric, and since the Cayley graph of $G$ is a metric space, by Proposition 2, $X$ is a quasi-metric space.

As an alternative proof strategy for Theorem 1, one might think to first directly prove that $X$ must be quasi-metric, before deducing that $G$ acts properly and cocompactly by isometries on the corresponding metric space constructed in the proof of Proposition 2 and applying the usual Švarc-Milnor lemma. However, there seems to be no easier way to prove that $X$ is quasi-metric than by establishing Theorem 1 .

Note that in the case $X$ is actually a geodesic metric space which is proper, so that our "metric" definitions of proper and cocompact actions agree with the usual topological definitions, we recover from Theorem 1 the original Švarc-Milnor lemma.

\section{DireCted Graphs AND SEMigroups as SemimetriC SPACES}

In this section we consider a particularly important class of semimetric spaces, namely those which arise from directed graphs.

By a (directed) graph $\Gamma$ we mean a set $R$ of vertices together with a set $E$ of edges and two functions $\iota: E \rightarrow R$ and $\tau: E \rightarrow R$ which describe respectively the initial vertex (or source) and the terminal vertex (or target) of each edge. Note that this definition permits loops and multiple edges, and places no cardinality restrictions on the vertex or edge sets.

It is easily verified that the vertex set of a directed graph $\Gamma$ is an example of a semimetric space, with the distance $d(x, y)$ between vertices $x$ and $y$ defined to be the infimum number of edges in a directed path from $x$ to $y$ ( $\infty$ if there is no path). For our purposes, it will also be convenient to regard a directed graph as a geodesic space in order to facilitate the application of continuous arguments. To this end, given a directed graph $\Gamma$ we define a new semimetric space $\Gamma^{*}$ with point set $\Gamma^{*}=R \cup(E \times(0,1))$ and metric defined as follows. If $x, y \in R$ are vertices in $\Gamma$, then $d(x, y)$ is the shortest length of a path from $x$ to $y$ in $\Gamma$. Otherwise we define

$$
\begin{gathered}
d((e, \mu), y)=(1-\mu)+d(\tau(e), y), \\
d(x,(e, \mu))=d(x, \iota(e))+\mu, \\
d((e, \mu),(f, \nu))= \begin{cases}\nu-\mu & \text { if } e=f \text { and } \nu \geq \mu, \\
d(\tau(e), \iota(f))+(1-\mu)+\nu & \text { otherwise. }\end{cases}
\end{gathered}
$$

The following elementary relation between $\Gamma$ and $\Gamma^{*}$ is now easily established.

Proposition 3. Let $\Gamma$ be a directed graph. Then $\Gamma^{*}$ is a geodesic semimetric space and the inclusion $\Gamma \subseteq \Gamma^{*}$ is an isometric embedding.

Notice that the inclusion of $\Gamma$ into $\Gamma^{*}$ is not in general a quasi-isometry. Since a typical point on an edge is "near" each of its endpoints in only one direction, there 
is no reason to suppose it lies in a strong ball about any vertex, let alone one of uniformly bounded radius. In fact, it is easy to show that the inclusion of $\Gamma$ into $\Gamma^{*}$ is a quasi-isometry if and only if $\Gamma$ (and hence $\Gamma^{*}$ by Corollary 1) is quasi-metric.

We now turn our attention to some semimetric spaces which arise naturally in semigroup theory. Let $S$ be a semigroup generated by a finite subset $A$. Then $S$ is naturally endowed with the structure of a semimetric space, with distance function defined by

$$
d_{A}(x, y)=\inf \left\{|w|: w \in A^{*}, x w=y\right\},
$$

where $A^{*}$ denotes the free monoid over $A$ and $|w|$ is the length of the word $w$. Note that $d_{A}(x, y)=\infty$ is possible since a semigroup can have proper right ideals. For $s \in S=\langle A\rangle$ we use $l_{A}(s)$ to denote the minimal length of a word over $A^{+}$that represents the element $s$.

A slightly more sophisticated semimetric space may be obtained by considering the (right) Cayley graph of $S$ with respect to $A$ which is the edge-labelled directed graph whose vertices are the elements of $S$ and with a directed edge from $x \in S$ to $y \in S$ labelled by $a \in A$ if and only if $x a=y$ in $S$. We denote by $\Gamma(S, A)$ the geodesic semimetric space obtained by applying the $*$-operation to this graph.

It follows from Proposition 3 that the natural inclusion of $S$ into $\Gamma(S, A)$ is an isometric embedding, but in general there is no reason to suppose it is a quasiisometry. Moreover, the semimetric space $\Gamma(S, A)$ carries more information than the semimetric space $\left(S, d_{A}\right)$, since it contains information about edge-multiplicities. So, in general, it is impossible to reconstruct $\Gamma(S, A)$ from $\left(S, d_{A}\right)$, while conversely $\left(S, d_{A}\right)$ can be obtained from $\Gamma(S, A)$ just by restricting it to the set of vertices.

There is also the obvious dual notion of a left Cayley graph which has the same vertex set but a different semimetric space. For finitely generated groups the corresponding left and right versions of each of these spaces are isometric, so nothing is lost by always working with just right Cayley graphs. For finitely generated semigroups the right and left Cayley graphs are not, in general, isometric. In fact, they may not even be quasi-isometric (as can be seen by taking a finitely generated semigroup with unequal numbers of $\mathcal{R}$-classes and $\mathcal{L}$-classes, in the sense to be defined in Section 5 below). From now on, unless otherwise stated, by the Cayley graph we shall always mean the right Cayley graph.

Proposition 4. Let $A$ and $B$ be finite generating sets for a semigroup $S$. Then $\left(S, d_{A}\right)$ is quasi-isometric to $\left(S, d_{B}\right)$.

Proof. Let $f$ be the identity mapping on $S$, viewed as a map from $\left(S, d_{B}\right)$ to $\left(S, d_{A}\right)$. Since $f$ is surjective its image is quasi-dense in $\left(S, d_{A}\right)$. Set $\lambda_{1}=\max \left\{l_{A}(b): b \in B\right\}$ and $\lambda_{2}=\max \left\{l_{B}(a): a \in A\right\}$. For all $x, y \in S$ it is easy to check by induction on $d_{B}(x, y)$ that $d_{A}(f(x), f(y)) \leq \lambda_{1} d_{B}(x, y)$. Similarly $d_{B}(x, y) \leq \lambda_{2} d_{A}(f(x), f(y))$. We conclude that $f$ is a $\left(\max \left\{\lambda_{1}, \lambda_{2}\right\}, 0,0\right)$-quasi-isometry.

However, in contrast to groups, if $A$ and $B$ are finite generating sets for a semigroup $S$, then $\Gamma(S, A)$ and $\Gamma(S, B)$ need not be quasi-isometric (see Example 1 below).

Definition 11. Let $S$ and $T$ be finitely generated semigroups with finite generating sets $A$ and $B$, respectively. We say that the semigroups $S$ and $T$ are (right) quasiisometric if $\left(S, d_{A}\right)$ and $\left(T, d_{B}\right)$ are quasi-isometric. 
Again, there is a dual notion of two semigroups being left quasi-isometric. Here we shall work only with right quasi-isometries between semigroups, and by quasiisometric we shall always mean right quasi-isometric. Note that as a result of the dependence on the choice of the generating set described above, we cannot use the right Cayley graph $\Gamma(S, A)$ in the above definition.

For a semigroup $S$ we use $S^{1}$ to denote the monoid $S \cup\{1\}$ where 1 is an adjoined identity, assumed to be disjoint from $S$. Let $S$ and $T$ be finitely generated semigroups. It is easy to see that $S$ and $T$ are quasi-isometric if and only if $S^{1}$ and $T^{1}$ are quasi-isometric. (For the less trivial of the two directions, one just has to observe that any quasi-isometry $f: S^{1} \rightarrow T^{1}$ must map $1_{S}$ to $1_{T}$ since these are the unique basepoints in the respective Cayley graphs.) In our discussions below there are various situations where we shall find it convenient to work with monoids rather than semigroups, and from this observation we see that no generality is lost in doing so.

Associated with any semimetric space $X$ is a natural preorder $\lesssim$ given by $x \lesssim y$ if and only if $d(x, y)<\infty$. Let $\sim$ denote the equivalence relation given by $x \sim y$ if and only if $x \lesssim y$ and $y \lesssim x$. We call the $\sim$-classes the strongly connected components of $X$. Given a semimetric space $X$, let $X / \sim$ denote the poset of equivalence classes of strongly connected components of $X$. The following proposition is an immediate consequence of the definition of quasi-isometry.

Proposition 5. Let $f: X \rightarrow Y$ be a quasi-isometry of semimetric spaces. Then $f$ maps each of the $\sim$-classes of $X$ quasi-isometrically into a $\sim$-class of $Y$, and this induces an isomorphism of partially ordered sets $(X / \sim) \rightarrow(Y / \sim)$.

Example 1. Let $S$ be the two element semigroup $\{a, 0\}$ where $a^{2}=0$ and $a 0=$ $0 a=0^{2}=0$. Then $A=\{a\}$ and $B=\{a, 0\}$ are both generating sets for $S$, but $\Gamma(S, A)$ and $\Gamma(S, B)$ are not quasi-isometric. Indeed, the poset associated to $\Gamma(S, A)$ has the property that any pair of points is comparable, while this is not the case in the poset associated with $\Gamma(S, B)$. Hence by Proposition 5 the spaces $\Gamma(S, A)$ and $\Gamma(S, B)$ are not quasi-isometric.

In particular it follows from Proposition 5 that if two semigroups $S$ and $T$ are quasi-isometric, then the partially ordered sets $S / \mathcal{R}$ and $T / \mathcal{R}$ must be isomorphic (where $\mathcal{R}$ denotes Green's $\mathcal{R}$-relation defined in Section 5 below).

A central question in geometric group theory is that of which algebraic properties are invariant under quasi-isometry. Such properties are called geometric, and wellknown examples include finiteness, the number of ends, having a free subgroup of finite index, being finitely presented, being hyperbolic, being automatic, being amenable, being accessible, the type of growth (see [8, p. 115, Section 50] and the references therein), having an abelian subgroup of finite index (see [5] and 29]), having a nilpotent subgroup of finite index (see [19]), being finitely presented with solvable word problem, and satisfying the homological finiteness condition $F_{n}$ or the condition $F P_{n}$ (see [2] and [3]).

It is natural to ask which properties are quasi-isometry invariants of semigroups. Certain properties, such as being finite, or having finitely many right ideals, are clearly quasi-isometry invariants (the latter observation follows from Proposition 5). We shall see below that several important properties of finitely generated semigroups are invariant under quasi-isometry. 


\section{SCHÜTZENBERGER GROUPS AND GRAPHS}

In this section we demonstrate how the theory developed in the preceding section can be applied to some problems in the theory of finitely generated semigroups and monoids. For any undefined concepts from semigroup theory we refer the reader to 22 .

The notions of a Schützenberger graph and a Schützenberger group lie at the heart of recent developments in geometric approaches in semigroup theory; see Steinberg [38, 39]. In [31] it was shown that under a certain finiteness assumption (see below) a Schützenberger group of a finitely generated monoid will be finitely generated. In fact, as was pointed out in [31, this result also follows from Schützenberger's original work [32, 33. In 39] a topological proof of the same result was given for the special case of maximal subgroups of inverse semigroups. Here, by considering the natural action of the Schützenberger group on its Schützenberger graph and applying the Švarc-Milnor lemma of Section 3 , we shall obtain an alternative proof of this result. At the same time we recover information relating the geometry of the Schützenberger graph to that of the Schützenberger group. This relationship is used below when we consider quasi-isometry invariants of semigroups.

First we must introduce some ideas and terminology from semigroup theory. Green's relations were introduced in [17, and ever since have played a fundamental role in the structure theory of semigroups. We give a brief overview of the theory here; for more details we refer the reader to 22 .

On any monoid $M$ we may define a pre-order $\leq_{\mathcal{R}}$ by $x \leq_{\mathcal{R}} y$ if and only if $x M \subseteq y M$. The relation $\mathcal{R}$ is defined to be the least equivalence relation containing $\leq_{\mathcal{R}}$, so $x \mathcal{R} y$ if and only if $x$ and $y$ generate the same principal right ideal. If $M$ is generated by $A$, then the $\mathcal{R}$-classes are the strongly connected components of $\left(M, d_{A}\right)$. A pre-order $\leq_{\mathcal{L}}$ and equivalence relation $\mathcal{L}$ can be defined in the obvious left-right dual way, and the intersection $\mathcal{R} \cap \mathcal{L}$ (which is also an equivalence relation) is denoted $\mathcal{H}$.

The importance of the $\mathcal{H}$-relation becomes apparent when considering the maximal subgroups of a monoid; those $\mathcal{H}$-classes which contain idempotents are exactly the maximal subgroups of the containing monoid. It is possible to associate a group to any $\mathcal{H}$-class of a monoid, which is called the Schützenberger group of the $\mathcal{H}$-class. If the $\mathcal{H}$-class happens to be a subgroup, then this group will be isomorphic to the Schützenberger group of the $\mathcal{H}$-class, so the notion of a Schützenberger group generalises that of a maximal subgroup. The (left) Schützenberger group is obtained by taking the action of the setwise stabiliser of $H$ on $H$, under left multiplication by elements of the monoid, and making it faithful. That is, given an arbitrary $\mathcal{H}$-class $H$ of $M$, let $\operatorname{Stab}(H)=\{s \in S: s H=H\}$ denote the (left) stabilizer of $H$ in $S$. Then define an equivalence relation $\sigma=\sigma(H)$ on the stabilizer by $(x, y) \in \sigma$ if and only if $x h=y h$ for all $h \in H$. It is straightforward to verify that $\sigma$ is a congruence and that $\mathcal{G}(H)=\operatorname{Stab}(H) / \sigma$ is a group, called the left Schützenberger group of $H$. One can also define the right Schützenberger group of $H$ in the natural way, and it turns out that the left and right Schützenberger groups are isomorphic to one another. For information about the basic properties of Schützenberger groups we refer the reader to [25, Section 2.3]. In particular, we recall here that the orbits of the action of $\mathcal{G}$ on an $\mathcal{R}$-class $R$ are precisely the $\mathcal{H}$-classes of $S$ contained in $R$ and 
that the action of $\mathcal{G}$ on such an $\mathcal{H}$-class is semiregular in the sense of permutation group theory, that is, that only the identity has a fixed point.

The Schützenberger graph $\Gamma(R, A)$ of $R$, with respect to $A$, is the strongly connected component of $h \in H$ in $\Gamma(M, A)$. It is easily seen to consist of those vertices which are elements of $R$, together with edges connecting them, and so can be obtained by beginning with a directed graph $\Delta$ with vertex set $R$ and a directed labelled edge from $x$ to $y$ labelled by $a \in A$ if and only if $x a=y$, and then setting $\Gamma(R, A)=\Delta^{*}$ (using the notation from Section 3). From its construction it is clear that for any generating set $A$ of $M, \Gamma(R, A)$ is a strongly connected geodesic semimetric space.

Now the group $\mathcal{G}(H)$ acts naturally on $R$ via $(s / \sigma) \cdot r=s r$. This action extends naturally to an action by isometries of $\mathcal{G}(H)$ on the Schützenberger graph $\Gamma(R, A)$. Thus, the group $\mathcal{G}(H)$ acts by isometries on the strongly connected geodesic semimetric space $\Gamma(R, A)$.

Theorem 2. Let $M$ be a monoid generated by a finite set $A$, let $H$ be an $\mathcal{H}$ class of $M$, let $G$ be the Schützenberger group of $H$, and let $\Gamma(R, A)$ denote the Schützenberger graph of the $\mathcal{R}$-class $R$ containing $H$. Then the left translation action of $G$ on $\Gamma(R, A)$ is outward proper and by isometries. The action is cocompact if and only if $R$ contains only finitely many $\mathcal{H}$-classes.

Proof. To show that the action is by isometries, it clearly suffices to show that the left translation action of $G$ on $R$ is by isometries. If $x, y \in R$ are such that $d(x, y)=n$, then there exist $a_{1}, \ldots, a_{n} \in A$ such that $x a_{1} \ldots a_{n}=y$. But then

$$
(s / \sigma) \cdot x a_{1} \ldots a_{n}=s x a_{1} \ldots a_{n}=s y=(s / \sigma) \cdot y
$$

so that $d((s / \sigma) \cdot x,(s / \sigma) \cdot y) \leq n=d(x, y)$. A similar argument using $(s / \sigma)^{-1}$ shows that $d((s / \sigma) \cdot x,(s / \sigma) \cdot y) \geq d(x, y)$.

To prove that the action is outward proper we must show that for each $x_{0} \in R$ and $0 \leq \epsilon<\infty$ the set

$$
Q=\left\{g \in G \mid g \overrightarrow{\mathcal{B}}_{\epsilon}\left(x_{0}\right) \cap \overrightarrow{\mathcal{B}}_{\epsilon}\left(x_{0}\right) \neq \varnothing\right\}
$$

is finite. Since $\Gamma(R, A)$ is strongly connected, it will clearly suffice to fix a basepoint $x_{0}=h \in H$ and prove the claim for $x_{0}$ and every $0 \leq \epsilon<\infty$.

Set $B=\overrightarrow{\mathcal{B}}_{\epsilon}\left(x_{0}\right)$, noting that $B$ contains only finitely many vertices of $\Gamma(R, A)$ since the out-degree of every vertex is bounded above by $|A|$. Let $\left\{x_{1}, \ldots, x_{n}\right\}$ be the set of vertices in $B$. Let $g \in Q$. By definition there exists $b \in B$ such that $g b \in B$. First suppose that $b$ is a vertex so that $b=x_{i}$ for some $i$. Since under the action of $G$ on $\Gamma(R, A)$ vertices are mapped to vertices, we have $g b=x_{j}$ for some $j$. But since $G$ acts on $R$ with trivial point stabilizers, it follows that $g$ is uniquely determined by the pair $\left(x_{i}, x_{j}\right)$, so it must be one of a fixed set of $n^{2}$ or fewer group elements.

If $b$ is not a vertex, say $b=(e, \mu)$, then consider the strong ball of radius $\epsilon+\mu$ based at $\iota(e)$ and apply the argument of the previous paragraph. This completes the proof that the action is outward proper.

For the second part of the theorem, suppose that $R$ is a union of finitely many $\mathcal{H}$-classes. Let $x_{0}=h \in H$. Since $\left(R, d_{A}\right)$ is strongly connected and $R$ is a union of finitely many $\mathcal{H}$-classes, it follows that there exists $\lambda \geq 0$ such that the strong ball $\mathcal{B}_{\lambda}\left(x_{0}\right)$ contains at least one vertex from every $\mathcal{H}$-class in $R$. We observed above 
that the orbits of the action of $G$ on $R$ are exactly the $\mathcal{H}$-classes, so it follows that the translates $\left(g \mathcal{B}_{\lambda}\left(x_{0}\right)\right)_{g \in G}$ cover $\Gamma(R, A)$. Hence, the action is cocompact.

For the converse, suppose that the action is cocompact. Then there is a strong ball $B$ of finite radius whose translates $(g B)_{g \in G}$ cover $\Gamma(R, A)$. Since $B$ has finite radius, it only intersects finitely many of the $\mathcal{H}$-classes in $R$. As the $\mathcal{H}$-classes of $R$ are the orbits under the action of $G$ on $R$, it follows that any vertex $h \in g B$ belongs to one of the finitely many $\mathcal{H}$-classes that $B$ intersects. Since $(g B)_{g \in G}$ covers $\Gamma(R, A)$ we conclude that $B$ intersects every $\mathcal{H}$-class in $R$, and thus $R$ is a union of finitely many $\mathcal{H}$-classes.

Theorems about finitely generated semigroups are often proved by technical, combinatorial means which, while convincing, yield relatively little insight into why the results hold. A case in point is a theorem of Ruskuc 31], originally proved using a Reidemeister-Schreier argument, which states that in a finitely generated monoid, if an $\mathcal{H}$-class $H$ lies in an $\mathcal{R}$-class containing only finitely many $\mathcal{H}$-classes, then the Schützenberger group of $H$ is finitely generated. Steinberg [39] has shown that this fact has a geometric interpretation in the special case of inverse semigroups. The following stronger statement arises as an immediate corollary of Theorems 1 and 2 , As well as being an alternative proof, we would argue that it also provides a more satisfactory explanation for this phenomenon.

Theorem 3. Let $M$ be a monoid generated by a finite set $A$, and let $H$ be an $\mathcal{H}$-class of $M$. If the $\mathcal{R}$-class $R$ that contains $H$ has only finitely many $\mathcal{H}$-classes, then the Schützenberger group $G$ of $H$ is finitely generated and quasi-isometric to the Schützenberger graph $\Gamma(R, A)$.

It follows from the comment after Proposition 3 that under the assumptions of Theorem $3 \mathcal{G}(H), \Gamma(R, A)$ and $\left(R, d_{A}\right)$ are all quasi-isometric to one another. Therefore, by the argument of Proposition 4 , if $R$ has only finitely many $\mathcal{H}$-classes, and $A$ and $B$ are any two finite generating sets for $M$, then the semimetric spaces $\Gamma(R, A)$ and $\Gamma(R, B)$ are quasi-isometric.

Theorem 3 is an example of a situation where one may apply the Švarc-Milnor lemma of Section 3 and thus avoid having to prove a Reidemeister-Schreier rewriting result. We also mention two other situations where Theorem 1 may be similarly applied.

In [40, Lemma 7.3], Steinberg proves (in the terminology defined in that paper) that if $S$ is a finitely generated inverse semigroup acting non-degenerately on a locally compact Hausdorff space $X$ and the orbit $\mathcal{O}$ of a point $x \in X$ is finite, then the isotropy group $G_{x}$ (defined in [40, Definition 7.1]) is itself finitely generated. As the author points out, the isotropy group $G_{x}$ acts naturally on the right of $L_{x}=d^{-1}(x)=\{[s, x]: s \in S\}$, where $[s, x]$ denotes the germ of $s$ at $x$ (see [40, Section 5.2]). The set $L_{x}$ plays the role of the $\mathcal{L}$-class in this setting. The set $L_{x}$ is the vertex set of a digraph (which is the analogue of the left Schützenberger graph), where there is a directed edge from $\left[s_{1}, x\right]$ to $\left[s_{2}, x\right]$ labelled by $\left[a, s_{1} x\right]$ when $s_{1}, s_{2} \in S$ satisfy $a s_{1}=s_{2}$. The action of the isotropy group $G_{x}$ on $L_{x}$ extends naturally to an action by isometries on this digraph, viewed as a semimetric space, and when the orbit $\mathcal{O}$ is finite the action is cocompact. Then, as in Theorem 3 above, one may deduce that the isotropy group $G_{x}$ is finitely generated as an application of Theorem 1 . 
In [16] Green's relations and Schützenberger groups are considered, but taken relative to a subsemigroup of a semigroup. Given a semigroup $S$ and a subsemigroup $T$ we write

$$
u \mathcal{R}^{T} v \Leftrightarrow u T^{1}=v T^{1}, \quad u \mathcal{L}^{T} v \Leftrightarrow T^{1} u=T^{1} v
$$

and $\mathcal{H}^{T}=\mathcal{R}^{T} \cap \mathcal{L}^{T}$. In [16] the Green index of $T$ in $S$ is defined to be one more than the number of $\mathcal{H}^{T}$-classes in $S \backslash T$. There are natural corresponding notions of $T$-relative Schützenberger groups and Schützenberger graphs. In this context, if $T$ is finitely generated by a set $A, R$ is an $\mathcal{R}^{T}$-class of $S$, and $H$ is an $\mathcal{H}^{T}$-class of $S$ contained in $R$, then the (left) $T$-relative Schützenberger group $\Gamma(H)$ of $H$ acts by isometries on the (right) $T$-relative Schützenberger graph $\Gamma(R, A)$, and this action is proper by [16, Proposition 5]. When $R$ is a union of finitely many $\mathcal{H}^{T}$-classes this action is cocompact and, just as in Theorem 3 above, it follows that $\Gamma(H)$ is finitely generated and quasi-isometric to $\Gamma(R, A)$. In particular, this shows that if $T$ is finitely generated and has finite Green index in $S$, then all of the relative Schützenberger groups of $\mathcal{H}^{T}$-classes in $S \backslash T$ are finitely generated.

In general, Schützenberger graphs can be very far away from being quasi-isometric to groups, as the following straightforward examples show.

Example 2. Let $T_{\mathbb{Z}}$ denote the full transformation monoid on the set $\mathbb{Z}$ of integers. The elements of $T_{\mathbb{Z}}$ are the maps from $\mathbb{Z}$ to itself, and multiplication is given by the usual composition of maps, where we view maps as acting on the right and compose from left to right. Let $\alpha$ and $\alpha^{-1}$ denote the infinite cycles given by

$$
n \alpha=n+1, \quad n \alpha^{-1}=n-1 \quad \text { for } n \in \mathbb{Z},
$$

and for $i \in \mathbb{Z}$ let $\gamma_{i}$ denote the constant mapping with image $i$. Set $A=\left\{\alpha, \alpha^{-1}, \gamma_{0}\right\}$ and define

$$
S=\langle A\rangle=\left\{\alpha^{n}: n \in \mathbb{Z}\right\} \cup\left\{\gamma_{i}: i \in \mathbb{Z}\right\} .
$$

The $\mathcal{R}$-class of $\gamma_{0}$ is the set of all constant maps $R=\left\{\gamma_{i}: i \in \mathbb{Z}\right\}$. We claim that the Schützenberger graph $\Gamma(R, A)$ is not quasi-metric. Indeed, for all $i \in \mathbb{Z}$, $d\left(\gamma_{n}, \gamma_{0}\right)=1$ (since $\left.\gamma_{n} \gamma_{0}=\gamma_{0}\right)$, while $d\left(\gamma_{0}, \gamma_{n}\right)=|n|$ (the unique shortest directed path from $\gamma_{0}$ to $\gamma_{n}$ is the one labelled by the word $\left.\alpha^{n}\right)$.

Also, in this example the Schützenberger group of $R$ is the trivial group, while the Schützenberger graph is infinite.

Example 3. Consider the bicyclic monoid $B$ defined by the finite presentation $\langle b, c \mid b c=1\rangle$ with respect to its usual generating set $A=\{b, c\}$. The Schützenberger graph $\Gamma\left(R_{1}, A\right)$ containing the identity element is a one-way infinite line with directed edges in both directions between adjacent vertices of the line. By the indegree of a vertex $v$ of the digraph $\Gamma\left(R_{1}, A\right)$ we mean the number of vertices $w$ such that there is a directed edge from $w$ to $v$. Dually we define the outdegree. In $\Gamma\left(R_{1}, A\right)$ all the vertices have finite indegree and outdegree. There is a unique vertex (namely the identity of $B$ ) with indegree and outdegree 1 , while all other vertices have indegree and outdegree 2 . The space $\Gamma\left(R_{1}, A\right)$ is quasi-metric, but it is not quasi-isometric to the corresponding Schützenberger group which is trivial.

Theorem 3 has some consequences regarding quasi-isometry invariants of finitely generated monoids.

Theorem 4. For finitely generated monoids with finitely many left and right ideals, finite presentability is a quasi-isometry invariant. 
Proof. Let $M, N$ be quasi-isometric finitely generated monoids, each with finitely many left and right ideals, and with finite generating sets $A, B$, respectively. Suppose that $M$ is finitely presented. We want to show that $N$ is finitely presented. Let $f:\left(N, d_{B}\right) \rightarrow\left(M, d_{A}\right)$ be a quasi-isometry. Let $R$ be an $\mathcal{R}$-class of $N$. By Proposition 5. $f(R)$ is contained in an $\mathcal{R}$-class, $K$ say, of $M$. Let $H \subseteq R$ be an $\mathcal{H}$-class of $N$, and let $U \subseteq K$ be an $\mathcal{H}$-class of $M$. Then by Theorem 3 and the transitivity of quasi-isometry we see that the semimetric spaces $\mathcal{G}(H),\left(R, d_{A}\right),\left(K, d_{B}\right)$ and $\mathcal{G}(U)$ are all quasi-isometric to one another. Thus the groups $\mathcal{G}(H)$ and $\mathcal{G}(U)$ are quasi-isometric. By assumption, $M$ is finitely presented, which by [31, Theorem 1.1] implies that $\mathcal{G}(U)$ is finitely presented. Since having a finite presentation is a quasi-isometry invariant for groups (see [8, p. 115, Section 50]), it follows that $\mathcal{G}(H)$ is finitely presented. As $H$ was an arbitrary $\mathcal{H}$-class of $N$ it follows that all Schützenberger groups of $N$ are finitely presented, which, along with the fact that $N$ has finitely many left and right ideals, by [31, Theorem 1.1] implies that $N$ is finitely presented.

For certain important classes of semigroups, finite generation is sufficient to guarantee that there are only finitely many $\mathcal{L}$ - and $\mathcal{R}$-classes, and so in these cases finite presentability is a quasi-isometry invariant.

Corollary 2. Finite presentability is a quasi-isometry invariant for finitely generated Clifford monoids and for finitely generated completely simple and completely 0-simple semigroups.

Note that although completely (0-)simple semigroups are not in general monoids, by the comment made after Definition 11, Theorem 4 can still be applied to them.

Question 1. Is finite presentability a quasi-isometry invariant of finitely generated semigroups in general?

A natural first step towards answering this question would be to first establish whether finite presentability is an isometry invariant of finitely generated semigroups. We finish the section with a few variants of Theorem 4

Theorem 5. For finitely generated monoids with finitely many left and right ideals, the property of being finite presented with solvable word problem is a quasi-isometry invariant.

Proof. Although not explicitly stated there, it is an easy consequence of the proof of [31, Theorem 3.2] that for a monoid $M$ with finitely many left and right ideals, $M$ is finitely presented with solvable word problem if and only if all of its Schützenberger groups are finitely presented with solvable word problem. Now the result follows by an argument identical to the one given in the proof of Theorem 4, along with the result of 2] stating that being finitely presented with solvable word problem is a quasi-isometry invariant of groups.

Recall that a semigroup is regular if every $\mathcal{R}$-class contains an idempotent. In [3] it was shown that the homological finiteness condition $F P_{n}$ is a quasi-isometry invariant of finitely generated groups. Along with the result in [15] for regular monoids with finitely many left and right ideals, and the result of [7] saying that for finitely presented groups the homotopical finiteness condition finite derivation type and $F P_{3}$ are equivalent, using the same argument as in the proof of Theorem 4 we obtain the following. For the definition of finite derivation type and more on its 
importance in the theory of string-rewriting systems and connections to the theory of diagram groups, see [20, 28].

Theorem 6. For finitely generated regular monoids with finitely many left and right ideals having finite derivation type is a quasi-isometry invariant.

Just as for Theorem 4, each of Theorems 5] and 6] apply to finitely generated Clifford monoids and completely 0 -simple semigroups.

\section{Growth}

In this section we study the relationship between quasi-isometry and the notion of growth in semigroups and monoids. Recall that a (discrete) growth function is a monotone non-decreasing function from $\mathbb{N}$ to $\mathbb{N}$. Growth functions for finitely generated groups were introduced independently by Milnor [26] and Švarc [41]. Since then the growth of both groups and monoids has become a subject of extensive study (see for example [4, 18, 34, 35, 36]). For growth functions $\alpha_{1}, \alpha_{2}$ we write $\alpha_{1} \preccurlyeq \alpha_{2}$ if there exist natural numbers $k_{1}, k_{2} \geq 1$ such that $\alpha_{1}(t) \leq k_{1} \alpha_{2}\left(k_{2} t\right)$ for all $t \in \mathbb{N}$. We define an equivalence relation on growth functions by $\alpha_{1} \sim \alpha_{2}$ if and only if $\alpha_{1} \preccurlyeq \alpha_{2}$ and $\alpha_{2} \preccurlyeq \alpha_{1}$. The $\sim$-class $[\alpha]$ of a growth function $\alpha$ is called the growth type or just growth of the function $\alpha$. For a semigroup $S$ generated by a finite set $A$ the function

$$
g_{S}: \mathbb{N} \rightarrow \mathbb{N}, \quad g_{S}(m)=\left|\left\{z \in S: l_{A}(z) \leq m\right\}\right|
$$

is called the growth function of the semigroup $S$ (where $l_{A}(z)$ denotes the length of the element $z$ as defined above). The growth type of this function is independent of the choice of the finite generating set and so is simply called the growth of the semigroup. See for example 35] for more about the growth of semigroups.

The following result follows from Definition 22 and Proposition 25 of [8, $\S \mathrm{VI}]$.

Lemma 2. Let $\alpha_{1}$ and $\alpha_{2}$ be growth functions. Then $\alpha_{1} \preccurlyeq \alpha_{2}$ if and only if there exist natural numbers $\lambda$ and $C$ such that

$$
\alpha_{1}(t) \leq \lambda \alpha_{2}(\lambda t+C)+C
$$

for all $t \in \mathbb{N}$.

Definition 12. A semimetric space $X$ is called uniformly quasi-locally bounded if

$$
\sup _{x \in X}\left|\overrightarrow{\mathcal{B}}_{t}(x)\right|<\infty
$$

for all $t \in \mathbb{N}$. For such a space $X$, the growth function of $X$ at $x_{0} \in X$ is the function

$$
\beta: \mathbb{N} \rightarrow \mathbb{N}, \quad t \mapsto\left|\overrightarrow{\mathcal{B}}_{t}\left(x_{0}\right)\right|
$$

Lemma 3. Let $X$ be a semimetric space that is uniformly quasi-locally bounded, and let $\beta_{0}$ and $\beta_{1}$ be the growth functions of $X$ at basepoints $x_{0}$ and $x_{1}$, respectively. Then $\beta_{0} \sim \beta_{1}$.

Proof. Since $x_{0}$ and $x_{1}$ are basepoints we have $d\left(x_{0}, x_{1}\right)<\infty$ and $d\left(x_{1}, x_{0}\right)<\infty$. Choose $C \in \mathbb{N}$ with $C>d\left(x_{1}, x_{0}\right)$. Now $\overrightarrow{\mathcal{B}}_{t}\left(x_{0}\right) \subseteq \overrightarrow{\mathcal{B}}_{t+C}\left(x_{1}\right)$, and hence $\beta_{0}(t) \leq$ $\beta_{1}(t+C)$ for all $t \in \mathbb{N}$. Therefore $\beta_{0} \preccurlyeq \beta_{1}$ by Lemma 2, A dual argument establishes $\beta_{1} \preccurlyeq \beta_{0}$. 
Let $S$ be a semigroup generated by a finite subset $A$. Then the semimetric space $X=\left(S^{1}, d_{A}\right)$ is uniformly quasi-locally bounded and, with respect to the basepoint 1 , the growth function of $X$ is the growth function of the semigroup $S$.

Proposition 6. Let $X_{1}$ and $X_{2}$ be uniformly quasi-locally bounded semimetric spaces with basepoints $x_{1}$ and $x_{2}$, respectively. Let $\beta_{j}$ denote the corresponding growth function for $j=1,2$. If $X_{1}$ quasi-isometrically embeds into $X_{2}$, then $\beta_{1} \preccurlyeq$ $\beta_{2}$. In particular, if $X_{1}$ and $X_{2}$ are quasi-isometric, then they have the same type of growth.

Proof. By Lemma 2 it will suffice to show that there exist natural numbers $\lambda$ and $C$ satisfying $\beta_{1}(t) \leq \lambda \beta_{2}(\lambda t+C)+C$ for all $t \in \mathbb{N}$.

Let $f: X_{1} \rightarrow X_{2}$ be a $(\lambda, \epsilon)$-quasi-isometric embedding. Clearly, we may assume without loss of generality that $\lambda$ and $\epsilon$ lie in $\mathbb{N}$. Since $x_{2}$ is a basepoint of $X_{2}$ it follows that $d_{2}\left(x_{2}, f\left(x_{1}\right)\right)<\infty$, and so we may choose a natural number $D$ with $D>d_{2}\left(x_{2}, f\left(x_{1}\right)\right)+\epsilon$. Now for any $x \in \overrightarrow{\mathcal{B}}_{t}\left(x_{1}\right)$ we have $d\left(x_{1}, x\right) \leq t$, and since $f$ is a $(\lambda, \epsilon)$-quasi-isometry it follows that $d\left(f\left(x_{1}\right), f(x)\right) \leq \lambda t+\epsilon$ and hence that

$$
d\left(x_{2}, f(x)\right) \leq d\left(x_{2}, f\left(x_{1}\right)\right)+\lambda t+\epsilon \leq \lambda t+D
$$

so that $f(x) \in \overrightarrow{\mathcal{B}}_{\lambda t+D}\left(x_{2}\right)$. Thus, $f\left(\overrightarrow{\mathcal{B}}_{t}\left(x_{1}\right)\right) \subseteq \overrightarrow{\mathcal{B}}_{\lambda t+D}\left(x_{2}\right)$, and so

$$
\left|f\left(\overrightarrow{\mathcal{B}}_{t}\left(x_{1}\right)\right)\right| \leq\left|\overrightarrow{\mathcal{B}}_{\lambda t+D}\left(x_{2}\right)\right|=\beta_{2}(\lambda t+D)
$$

for all $t \in \mathbb{N}$. Next consider the fibres of the mapping $f$. For any $z \in X_{2}$ and any $x, y \in f^{-1}(z)$ we have $d_{1}(x, y) \leq \lambda \epsilon$. Since $X_{1}$ is uniformly quasi-locally bounded there is a natural number $E$ such that

$$
\sup _{z \in X_{2}}\left|f^{-1}(z)\right| \leq E \text {. }
$$

Therefore

$$
\left|f\left(\overrightarrow{\mathcal{B}}\left(x_{1}, t\right)\right)\right| \geq\left|\overrightarrow{\mathcal{B}}\left(x_{1}, t\right)\right| / E
$$

and combining this with the previous formula we conclude that

$$
\beta_{1}(t)=\left|\overrightarrow{\mathcal{B}}\left(x_{1}, t\right)\right| \leq E\left|f\left(\overrightarrow{\mathcal{B}}\left(x_{1}, t\right)\right)\right| \leq E \beta_{2}(\lambda t+D)
$$

for all $t \in \mathbb{N}$. So setting $\mu=\max (E, \lambda) \in \mathbb{N}$ we obtain

$$
\beta_{1}(t) \leq \mu \beta_{2}(\mu t+D) \leq \mu \beta_{2}(\mu t+D)+D
$$

for all $t \in \mathbb{N}$, as required.

Let $S$ be a finitely generated semigroup. If $A$ and $B$ are two finite generating sets for $S$, then by Lemma 4 the semimetric spaces $\left(S, d_{A}\right)$ and $\left(S, d_{B}\right)$ are quasiisometric, and so by Proposition [6 the corresponding growth functions are of the same type. Thus (as mentioned above) the growth type depends only on the semigroup and not on the choice of the generating set. More generally we have the following consequence of Proposition 6 .

Theorem 7. Let $S_{1}$ and $S_{2}$ be finitely generated semigroups with finite generating sets $A_{1}, A_{2}$, and let $\beta_{1}, \beta_{2}$ be the corresponding growth functions. If $\left(S_{1}, d_{A_{1}}\right)$ and $\left(S_{2}, d_{A_{2}}\right)$ quasi-isometrically embed into each other, then $S_{1}$ and $S_{2}$ have the same growth type. In particular, growth type is a quasi-isometry invariant for finitely generated semigroups. 
One application of Theorem 7 is to provide examples of pairs of semigroups which are not quasi-isometric. For example, finitely generated free commutative monoids of different rank are not quasi-isometric, since the free commutative monoid on $d$ generators has polynomial growth rate of order $d$.

\section{ENDS}

In this section we study the relationship between quasi-isometry and the number of ends of a monoid. The concept of ends goes back to the work of Freudenthal [12, 13], who introduced the notion in order to try to capture the idea of connectivity at infinity for a topological space. Hopf 21] showed that if the translates of a compact set under the action of a group of homeomorphisms cover the whole space, then the space has one, two or infinitely many (in fact, uncountably many) ends. In particular, a finitely generated group has one, two or infinitely many ends (since it acts transitively on its Cayley graph), and the celebrated result of Stallings [37. states that a finitely generated group has more than one end if and only if it admits a non-trivial decomposition as an amalgamated free product or an HNN extension over a finite subgroup. For finitely generated groups (and more generally for locally finite undirected graphs [27, Proposition 1]) it is well known that the number of ends is a quasi-isometry invariant. See 9] for more on the importance of ends in group theory.

Jackson and Kilibarda 23. have recently initiated the study of ends of finitely generated monoids. They define the number of ends of a monoid $M$ to be the supremum number of infinite connected components that may be obtained by removing a finite set of vertices from the underlying undirected graph of the right Cayley graph of $M$. In this section we show that the number of ends of a monoid, defined in this way, is a quasi-isometry invariant. We first need a preliminary lemma.

Lemma 4. Let $f: M \rightarrow N$ be a quasi-isometric embedding of finitely generated monoids and $S \subseteq M$ a subset of $M$. If $S$ is infinite, then $f(S)$ is infinite.

Proof. Suppose this is false for a contradiction. Then by the pigeon hole principle there exists an infinite subset $T \subseteq S$ and a point $n \in N$ such that $f(T)=\{n\}$. Now since the Cayley graph of $M$ has finite outdegree, $T$ must contain elements $t$ such that $d\left(1_{M}, t\right)$ is finite but arbitrarily large. But $d\left(f\left(1_{M}\right), f(t)\right)=d\left(f\left(1_{M}\right), n\right)$ is constant as $t$ varies within $T$, which clearly contradicts the assumption that $f$ is a quasi-isometric embedding.

Theorem 8. Let $(M, X)$ and $(N, Y)$ be quasi-isometric finitely generated monoids. Then $(M, X)$ and $(N, Y)$ have the same number of ends.

Proof. Let $f: M \rightarrow N$ be a $(\lambda, \epsilon, \mu)$-quasi-isometry.

Suppose $S \subseteq M$ is a finite subset separating the Cayley graph of $M$ into $r$ infinite components $C_{1}, \ldots, C_{r}$. By [23, Lemma 5], $S$ separates $M$ into only finitely many components in total. It follows that we may absorb any finite components into $S$, and assume without loss of generality that

$$
M=S \cup C_{1} \cup \cdots \cup C_{r} .
$$

Let $\omega=\lambda^{2}(2 \mu+\epsilon+1)+\epsilon$ and define

$$
\begin{aligned}
& T=\{t \in N \mid d(f(s), t) \leq \omega \text { for some } s \in S\} \text { and } \\
& U=\{t \in N \mid d(f(s), t) \leq \omega+\mu \text { for some } s \in S\} .
\end{aligned}
$$


Notice that $T$ and $U$ are finite, because $S$ is finite and the outdegree of each vertex in the Cayley graph of $N$ is finite. For $1 \leq i \leq r$ define $D_{i}=f\left(C_{i}\right) \backslash T$, and $E_{i}=f\left(C_{i}\right) \backslash U$. We claim that $U$ separates $N$ into components, at least $r$ of which are infinite.

We first claim that if $p \neq q$, there are no directed paths of length less than $2 \mu+1$ from $D_{p}$ to $D_{q}$. Indeed, suppose for a contradiction that there was such a path. Since $D_{p} \subseteq f\left(C_{p}\right)$ and $D_{q} \subseteq f\left(C_{q}\right)$ we may assume the path runs from $f(r) \in D_{p}$ to $f(s) \in D_{q}$ where $r \in C_{p}$ and $s \in C_{q}$. Now since $f$ is a $(\lambda, \epsilon, \mu)$-quasi-isometry, there is a directed path of length at most $\lambda(2 \mu+1+\epsilon)$ from $r$ to $s$. By the assumption on $S$, this path must pass through $S$; let $t \in S$ be a vertex on it. Then there is a directed path from $t$ to $s$ of length at most $\lambda(2 \mu+1+\epsilon)$, so once again using the fact that $f$ is a $(\lambda, \epsilon, \mu)$-quasi-isometry, there is a directed path from $f(t) \in f(S)$ to $f(s)$ of length at most $\lambda[\lambda(2 \mu+1+\epsilon)]+\epsilon=\omega$. But by the definition of $T$, this means that $f(s) \in T$, which contradicts the claim that $f(s) \in D_{q}=C_{q} \backslash T$.

Next, we claim that for any $i, j \in\{1, \ldots, k\}$ with $i \neq j$, every undirected path connecting a vertex in $D_{i}$ to a vertex in $D_{j}$ passes through $U$. Indeed, suppose for a contradiction that $\pi$ is an undirected path from some $D_{i}$ to some $D_{j}$ which does not pass through $U$. We shall show that there is a directed path of length at most $2 \mu+1$ from some $D_{p}$ to some $D_{q}$ (with $p \neq q$ ), thus contradicting the previous claim. Since $\pi$ is a path between vertices in a graph, we may assume it has integer length. First, if $\pi$ has length 1 , then it is a directed path (either from $D_{i}$ to $D_{j}$ or from $D_{j}$ to $D_{i}$ ) and so clearly has the required form. Next, if the final vertex of $\pi$ lies within a strong ball of radius $\mu$ around $D_{i}$, then clearly there is a path of length $\mu$ from $D_{i}$ to $D_{j}$, so the claim again holds.

Otherwise, let $x$ be the first vertex on the path $\pi$ which does not lie within a strong ball of radius $\mu$ around $D_{i}$, and let $w$ be the immediately preceding vertex. Then there is either an edge from $x$ to $w$ or an edge from $w$ to $x$. Since $f$ is a $(\lambda, \epsilon, \mu)$-quasi-isometry, $f(M)$ is $\mu$-quasi-dense in $N$, so $x$ lies within a strong ball of radius $\mu$ around $f(y)$ for some $y \in M$. Since $M=S \cup C_{1} \cup \cdots \cup C_{r}$ we must have $y \in S$ or $y \in C_{k}$ for some $k$. If $y$ were in $S$, then, since $\mu \leq \omega$, by the definition of $T$ we would have $x \in T \subseteq U$, contradicting the assumption on $\pi$. So instead we must have $y \in C_{k}$ for some $k$. Now if $f(y) \in T$, then since $d(f(y), x) \leq \mu$ we would again have $x \in U$, once more contradicting the assumption on $\pi$. There remains only the possibility that $f(y) \in D_{k}$. Moreover, since $x$ was chosen not to be within a strong ball of radius $\mu$ around $D_{i}$, we have $k \neq i$.

Now suppose there is an edge from $x$ to $w$. Since $x$ and $w$ are within strong balls of radius $\mu$ around $D_{k}$ and $D_{i}$, respectively, there is a directed path of length at most $2 \mu+1$ from $D_{k}$ to $D_{i}$, which is a contradiction. On the other hand, if there is an edge from $w$ to $x$, then by the same argument there is a directed path of length at most $2 \mu+1$ from $D_{i}$ to $D_{k}$, which is again a contradiction.

We have now shown that every undirected path connecting $D_{i}$ to $D_{j}$ with $i \neq j$ passes through $U$. In particular, since $E_{i} \subseteq D_{i}$ for each $i$, every undirected path from $E_{i}$ to $E_{j}$ passes through $U$. To complete the proof that $T$ separates $N$ into at least $r$ infinite components, it will suffice to show that each $E_{i}$ contains an infinite set of vertices which remain connected when $U$ is removed. By [23, Lemma 5] again, $U$ separates $N$ into only finitely many components. Since it separates $E_{i}$ from everything else, it follows that it separates $E_{i}$ into only finitely many components. 
But $C_{i}$ is infinite, so Lemma 4 ensures that $f\left(C_{i}\right)$ is infinite, and since $U$ is finite it follows that $E_{i}=f\left(C_{i}\right) \backslash U$ is infinite, as required.

We have now shown that if $M$ can be separated into $r$ infinite components, then $N$ can be separated into at least $r$ infinite components. It follows that if $M$ has $r$ ends, then $N$ has at least $r$ ends, while if $M$ has infinitely many ends, then so does $N$. By symmetry of assumption, it follows that $M$ and $N$ have the same number of ends.

Combining Theorem 8 with Proposition 4, we recover the following important fact, which was first proved in 23 ] by an entirely different combinatorial argument.

Corollary 3. The number of ends of a finitely generated monoid is independent of the choice of a finite generating set.

As mentioned above, it is well known that every Cayley graph of a finitely generated group has one, two or infinitely many ends. Jackson and Kilibarda 23 . showed that the corresponding statement for monoids does not hold. However, as a corollary of Theorem 3 , we see that in the case of $\mathcal{R}$-classes with finitely many $\mathcal{H}$-classes, a corresponding statement does hold for Schützenberger graphs, where the number of ends of the Schützenberger graph of an $\mathcal{R}$-class $R$ is defined as the number of ends of the graph obtained by restricting the undirected Cayley graph of the monoid to the set of vertices $R$.

Corollary 4. Let $M$ be a finitely generated monoid and let $R$ be an $\mathcal{R}$-class of $M$. If $R$ is a union of finitely many $\mathcal{H}$-classes, then the Schützenberger graph of $R$ has 1, 2 or infinitely many ends.

Proof. Let $A$ be a finite generating set for $M$ and let $H$ be an $\mathcal{H}$-class of $M$ in $R$. By Theorem 3 the Schützenberger graph $\Gamma(R, A)$ is quasi-isometric to the Schützenberger group $G$ of $H$. By the comment immediately following Proposition 3. since $\Gamma(R, A)$ is quasi-metric it follows that $\Gamma(R, A)$ is quasi-isometric to $\left(R, d_{A}\right)$. Since $\left(R, d_{A}\right)$ is quasi-metric it is easily deduced that, viewed as a directed graph, $\left(R, d_{A}\right)$ is quasi-isometric to its underlying undirected graph $\Delta$. This implies that $\Delta$ is quasi-isometric to the finitely generated group $G$. Therefore $\Delta$ is locally-finite and has the same number of ends as $G$ by [27, Proposition 1]. We conclude that the Schützenberger graph of $R$ has the same number of ends as the Schützenberger group $G$, and thus in particular has 1, 2 or infinitely many ends.

\section{ExAmples}

In this section we give some examples of monoids that are quasi-isometric to one another. The idea, roughly speaking, is that for two monoids to be quasi-isometric, globally they must share the same $\mathcal{R}$-class poset structure and locally they must have group structure that is the same up to quasi-isometry. Recall that a semigroup is called right simple if it has a single $\mathcal{R}$-class.

Proposition 7. The property of being a group is a quasi-isometry invariant of finitely generated monoids.

Proof. Let $M$ be a finitely generated monoid that is quasi-isometric to a finitely generated group $G$. Since the number of $\mathcal{R}$-classes is a quasi-isometry invariant, this implies that $M$ is right simple (i.e. has a single $\mathcal{R}$-class). But any right simple monoid is necessarily a group (see [6, Theorem 1.27]). 
Proposition 8. A finitely generated semigroup is right simple if and only if it is quasi-metric.

Proof. Let $S$ be a semigroup generated by a finite set $A \subseteq S$. If $S$ is quasi-metric, then $\left(S, d_{A}\right)$ is strongly connected and hence is right simple.

Conversely, suppose that $S$ is right simple. Define

$$
\lambda=\max \left\{d_{A}(b a, b): a, b \in A\right\}
$$

which exists since $A$ is finite and is finite since $S$ is right simple. Then for all $x \in S=\langle A\rangle$ and $a \in A$, writing $x=x^{\prime} b$ where $b \in A$, we obtain

$$
d_{A}(x a, x) \leq d_{A}(b a, b) \leq \lambda .
$$

It follows that for all $x, y \in S$

$$
d(y, x) \leq \lambda d(x, y)
$$

and therefore $\left(S, d_{A}\right)$ is quasi-metric.

It is well known, and follows quite easily from the Švarc-Milnor lemma, that a surjective morphism of finitely generated groups is a quasi-isometry if and only if it has finite kernel. The natural extension of this fact to monoids is the following.

Proposition 9. Let $S$ be a semigroup generated by a finite subset $A$, and let $\eta$ be a congruence on $S$. Then the natural map $\phi: S \rightarrow S / \eta$ sending $s \mapsto s / \eta$ is a quasi-isometry if and only if there is a bound on the $d_{A}$-diameter of the $\eta$-classes of $S$.

Proof. First suppose that there is a bound, say $R>0$, on the $d_{A}$-diameter of the $\eta$-classes of $S$. We claim that the map $\phi: S \rightarrow S / \eta$ given by $s \mapsto s / \eta$ is a $(\lambda, \epsilon, \mu)$-quasi-isometry where $\lambda=1, \epsilon=R$ and $\mu=0$.

The mapping $\phi$ is surjective, and hence $\phi(S)$ is 0-quasi-dense in $S / \eta$.

Let $x, y \in S$. If $d_{A}(x, y)=r$, then we can write $y=x a_{1} \cdots a_{r}$, where $a_{i} \in A$ for all $i$, and so

$$
(x / \eta)\left(a_{1} / \eta\right) \cdots\left(a_{r} / \eta\right)=y / \eta,
$$

which implies $d_{A / \eta}(\phi(x), \phi(y)) \leq r=d(x, y)$.

Also, if $d_{A / \eta}(f(x), f(y))=k$, then this means that we can write

$$
(x / \eta)\left(a_{1} / \eta\right) \cdots\left(a_{k} / \eta\right)=(y / \eta)
$$

where $a_{i} \in A$ for all $i$. This implies $\left(x a_{1} \cdots a_{k}, y\right) \in \eta$, and so $d_{A}(x, y) \leq k+R=$ $d_{A / \eta}(f(x), f(y))+R$. We conclude that for all $x, y \in S$,

$$
d_{A}(x, y)-R \leq d_{A / \eta}(f(x), f(y)) \leq d_{A}(x, y),
$$

as required.

For the converse suppose that $\phi$ is a quasi-isometry, say with constants $(\lambda, \epsilon, \mu)$. Let $x, y \in S$ with $(x, y) \in \eta$. Then $\phi(x)=\phi(y)$, so

$$
\frac{1}{\lambda} d_{A}(x, y)-\epsilon \leq d_{A / \eta}(\phi(x), \phi(y))=0,
$$

which implies $d_{A}(x, y) \leq \epsilon \lambda$. Thus $\epsilon \lambda$ is an upper bound on the $d_{A}$-diameter of the $\eta$-classes of $S$. 
Note that it does not suffice for the above result to require that the cardinalities of $\eta$-classes be bounded, let alone merely that they be finite. For example, if $S$ is any finite monoid with more than one $\mathcal{R}$-class, then the homomorphism from $S$ onto the trivial monoid satisfies these conditions, but $S$ is not quasi-isometric to the trivial monoid.

A particular instance where the conditions of the above proposition are satisfied is when $S \cong M \times G$, where $G$ is a finite group, $M$ is a monoid and $\eta$ is the congruence corresponding to the natural projection $M \times G \rightarrow M,(m, g) \mapsto m$.

Corollary 5. Let $M$ be a finitely generated monoid, and let $G$ be a finite group. Then $M \times G$ is quasi-isometric to $M$.

In fact, for the same reason this result holds more generally for the semidirect product of a monoid and a group (with the monoid acting on the group by endomorphisms).

If $G$ is a finitely generated group and $H$ is a subgroup of $G$ of finite index, then, as a standard application of the Švarc-Milnor lemma, $H$ is finitely generated and is quasi-isometric to $G$. This fact may be used to give further examples of quasi-isometric monoids.

Proposition 10. Let $S=M^{0}[G ; I, \Lambda, P]$ be a finitely generated completely 0 -simple semigroup represented as a 0-Rees matrix semigroup over a group. Let $H$ be a subgroup of $G$ and suppose that every non-zero entry of $P$ belongs to $H$. If $H$ has finite index in $G$, then $S$ and $T=M^{0}[H ; I, \Lambda, P]$ are quasi-isometric.

\section{REFERENCES}

[1] Algebraic theory of machines, languages, and semigroups. Edited by M. A. Arbib. With a major contribution by K. Krohn and J. L. Rhodes. Academic Press, New York, 1968. MR0232875 (38:1198)

[2] J. M. Alonso. Inégalités isopérimétriques et quasi-isométries. C. R. Acad. Sci. Paris Sér. I Math., 311(12):761-764, 1990. MR.1082628 (91k:57004)

[3] J. M. Alonso. Finiteness conditions on groups and quasi-isometries. J. Pure Appl. Algebra, 95(2):121-129, 1994. MR.1293049 (95f:20083)

[4] G. M. Bergman. A note on growth functions of algebras and semigroups. Technical report, Department of Mathematics, University of California, Berkeley, 1978.

[5] M. R. Bridson and S. M. Gersten. The optimal isoperimetric inequality for torus bundles over the circle. Quart. J. Math. Oxford Ser. (2), 47(185):1-23, 1996. MR.1380947 (97c:20047)

[6] A. H. Clifford and G. B. Preston. The algebraic theory of semigroups. Vol. I, Mathematical Surveys, No. 7. American Mathematical Society, Providence, R.I. 1961. MR0132791 (24:A2627)

[7] R. Cremanns and F. Otto. For groups the property of having finite derivation type is equivalent to the homological finiteness condition $\mathrm{FP}_{3}$. J. Symbolic Comput., 22(2):155-177, 1996. MR 1422144 (98b:20081)

[8] P. de la Harpe. Topics in geometric group theory. Chicago Lectures in Mathematics. University of Chicago Press, Chicago, IL, 2000. MR.1786869 (2001i:20081)

[9] W. Dicks and M. J. Dunwoody. Groups acting on graphs, volume 17 of Cambridge Studies in Advanced Mathematics. Cambridge University Press, Cambridge, 1989. MR1001965 (91b:20001)

[10] S. Eilenberg. Automata, languages, and machines. Vol. A. Academic Press [A subsidiary of Harcourt Brace Jovanovich, Publishers], New York, 1974. Pure and Applied Mathematics, Vol. 58. MR0530382 (58:26604a)

[11] S. Eilenberg. Automata, languages, and machines. Vol. B. Academic Press [Harcourt Brace Jovanovich Publishers], New York, 1976. With two chapters ("Depth decomposition theorem" and "Complexity of semigroups and morphisms") by B. Tilson, Pure and Applied Mathematics, Vol. 59. 
[12] H. Freudenthal. Über die Enden topologischer Räume und Gruppen. Math. Z., 33(1):692-713, 1931. MR 1545233

[13] H. Freudenthal. Neuaufbau der Endentheorie. Ann. of Math. (2), 43:261-279, 1942. MR0006504 (3:315a)

[14] É. Ghys and P. de la Harpe. Infinite groups as geometric objects (after Gromov). In Ergodic theory, symbolic dynamics, and hyperbolic spaces (Trieste, 1989), Oxford Sci. Publ., pages 299-314. Oxford Univ. Press, New York, 1991.

[15] R. Gray and A. Malheiro. Homotopy bases and finite derivation type for subgroups of monoids. Preprint at arXiv:math.GR/0912.1284.

[16] R. Gray and N. Ruškuc. Green index and finiteness conditions for semigroups. J. Algebra, 320(8):3145-3164, 2008. MR2450719 (2009h:20065)

[17] J. A. Green. On the structure of semigroups. Ann. of Math. (2), 54:163-172, 1951. MR0042380 (13:100d)

[18] R. I. Grigorchuk. Semigroups with cancellations of degree growth. Mat. Zametki, 43(3):305319, 428, 1988. MR941053 (89f:20065)

[19] M. Gromov. Groups of polynomial growth and expanding maps. Inst. Hautes Études Sci. Publ. Math., (53):53-73, 1981. MR623534(83b:53041)

[20] V. Guba and M. Sapir. Diagram groups. Mem. Amer. Math. Soc., 130(620):viii+117, 1997. MR.1396957 (98f:20013)

[21] H. Hopf. Enden offener Räume und unendliche diskontinuierliche Gruppen. Comment. Math. Helv., 16:81-100, 1944. MR0010267 (5:272e)

[22] J. M. Howie. Fundamentals of semigroup theory, volume 12 of London Mathematical Society Monographs. New Series. The Clarendon Press, Oxford University Press, New York, 1995. Oxford Science Publications. MR1455373 (98e:20059)

[23] D. A. Jackson and V. Kilibarda. Ends for monoids and semigroups. J. Aust. Math. Soc., 87(1):101-127, 2009. MR2538640(2010h:20153)

[24] J. C. Kelly. Bitopological spaces. Proc. London Math. Soc. (3), 13:71-89, 1963. MR0143169 $(26: 729)$

[25] G. Lallement. Semigroups and combinatorial applications. John Wiley \& Sons, New YorkChichester-Brisbane, 1979. Pure and Applied Mathematics, A Wiley-Interscience Publication. MR.530552(81j:20082)

[26] J. Milnor. A note on curvature and fundamental group. J. Differential Geometry, 2:1-7, 1968. MR0232311(38:636)

[27] R. G. Möller. Ends of graphs. II. Math. Proc. Cambridge Philos. Soc., 111(3):455-460, 1992. MR $1151324(93 a: 05067)$

[28] F. Otto and Y. Kobayashi. Properties of monoids that are presented by finite convergent string-rewriting systems - a survey. In Advances in algorithms, languages, and complexity, pages 225-266. Kluwer Acad. Publ., Dordrecht, 1997. MR1447453 (98f:68148)

[29] P. Pansu. Croissance des boules et des géodésiques fermées dans les nilvariétés. Ergodic Theory Dynam. Systems, 3(3):415-445, 1983. MR741395 (85m:53040)

[30] J. Richter-Gebert, B. Sturmfels, and T. Theobald. First steps in tropical geometry. In Idempotent mathematics and mathematical physics, volume 377 of Contemp. Math., pages 289-317. Amer. Math. Soc., Providence, RI, 2005. MR2149011 (2006d:14073)

[31] N. Ruškuc. On finite presentability of monoids and their Schützenberger groups. Pacific J. Math., 195(2):487-509, 2000. MR1782175 (2001f:20127)

[32] M. P. Schützenberger. D-représentation des demi-groupes. C. R. Acad. Sci. Paris, 244:19941996, 1957. MR0086820 (19:249b)

[33] M. P. Schützenberger. Sur la représentation monomiale des demi-groupes. C. R. Acad. Sci. Paris, 246:865-867, 1958. MR0095886(20:2384)

[34] L. M. Shneerson. Relatively free semigroups of intermediate growth. J. Algebra, 235(2):484546, 2001. MR1805469 (2002a:20068)

[35] L. M. Shneerson. Types of growth and identities of semigroups. Internat. J. Algebra Comput., 15(5-6):1189-1204, 2005. MR2197827 (2006j:20086)

[36] L. M. Shneerson. Polynomial growth in semigroup varieties. J. Algebra, 320(6):2218-2279, 2008. MR2437499 (2009d:20139)

[37] J. R. Stallings. On torsion-free groups with infinitely many ends. Ann. of Math. (2), 88:312334, 1968. MR0228573(37:4153) 
[38] B. Steinberg. Finite state automata: A geometric approach. Trans. Amer. Math. Soc., 353(9):3409-3464 (electronic), 2001. MR:1837243 (2002c:20106)

[39] B. Steinberg. A topological approach to inverse and regular semigroups. Pacific J. Math., 208(2):367-396, 2003. MR1971670 (2004k:20130)

[40] B. Steinberg. A groupoid approach to discrete inverse semigroup algebras. Adv. Math., 223(2):689-727, 2010. MR2565546 (2010k:20113)

[41] A. S. Švarc. A volume invariant of coverings. Dokl. Akad. Nauk SSSR (N.S.), 105:32-34, 1955. MR0075634(17:781d)

[42] W. A. Wilson. On Quasi-Metric Spaces. Amer. J. Math., 53(3):675-684, 1931. MR1506845

School of Mathematics and Statistics, University of St Andrews, St Andrews KY16 9SS, SCOTLAND

Current address: CAUL Universidade de Lisboa, Av. Prof. Gama Pinto, 2, 1649-003 Lisboa, Portugal

E-mail address: rdgray@fc.ul.pt

School of Mathematics, University of Manchester, Manchester M13 9PL, England

E-mail address: Mark.Kambites@manchester.ac.uk 